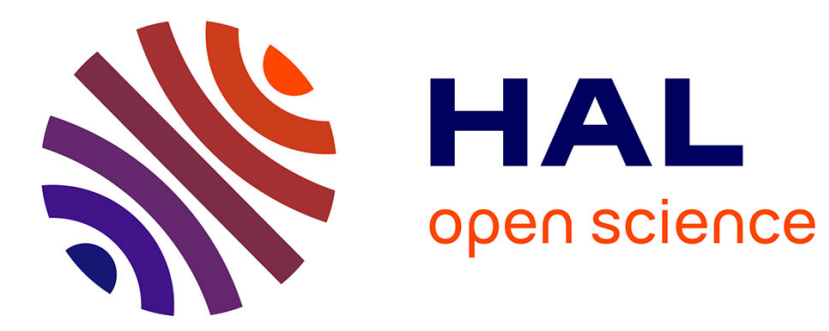

\title{
Probing the 3D structure of cornea-like collagen liquid crystals with polarization-resolved SHG microscopy
}

Claire Teulon, Aurélien Tidu, François Portier, Gervaise Mosser, Marie-Claire Schanne-Klein

\section{- To cite this version:}

Claire Teulon, Aurélien Tidu, François Portier, Gervaise Mosser, Marie-Claire Schanne-Klein. Probing the 3D structure of cornea-like collagen liquid crystals with polarization-resolved SHG microscopy. Optics Express, 2016, 24, pp.16084 - 10. 10.1364/OE.24.016084.v001 . hal-01421625

HAL Id: hal-01421625

https: / hal-polytechnique.archives-ouvertes.fr/hal-01421625

Submitted on 22 Dec 2016

HAL is a multi-disciplinary open access archive for the deposit and dissemination of scientific research documents, whether they are published or not. The documents may come from teaching and research institutions in France or abroad, or from public or private research centers.
L'archive ouverte pluridisciplinaire HAL, est destinée au dépôt et à la diffusion de documents scientifiques de niveau recherche, publiés ou non, émanant des établissements d'enseignement et de recherche français ou étrangers, des laboratoires publics ou privés. 


\title{
Probing the 3D structure of cornea-like collagen liquid crystals with polarization-resolved SHG microscopy
}

\author{
Claire Teulon, ${ }^{1}$ Aurélien Tidu, ${ }^{2}$ François Portier, ${ }^{2}$ \\ Gervaise Mosser, ${ }^{2}$ And MARIE-Claire SChanne-KLeIn ${ }^{1, *}$ \\ ${ }^{1}$ LOB, Ecole Polytechnique, CNRS, Inserm U1182, Université Paris-Saclay, F-91128 Palaiseau, France \\ ${ }^{2}$ Sorbonne Universités, UPMC Univ Paris 06, CNRS, Collège de France, LCMCP, F-75005 Paris, France \\ *marie-claire.schanne-klein@polytechnique.edu
}

\begin{abstract}
This work aims at characterizing the three-dimensional organization of liquid crystals composed of collagen, in order to determine the physico-chemical conditions leading to highly organized structures found in biological tissues such as cornea. To that end, we use secondharmonic generation (SHG) microscopy, since aligned collagen structures have been shown to exhibit intrinsic SHG signals. We combine polarization-resolved SHG experiments (P-SHG) with the theoretical derivation of the SHG signal of collagen molecules tilted with respect to the focal plane. Our P-SHG images exhibit striated patterns with variable contrast, as expected from our analytical and numerical calculations for plywood-like nematic structures similar to the ones found in the cornea. This study demonstrates the benefits of P-SHG microscopy for in situ characterization of highly organized biopolymers at micrometer scale, and the unique sensitivity of this nonlinear optical technique to the orientation of collagen molecules.
\end{abstract}

(C) 2016 Optical Society of America

OCIS codes: (180.4315) Nonlinear microscopy; (190.2620) Harmonic generation and mixing; (120.5410) Polarimetry; (170.3660) Light propagation in tissues; (170.6935) Tissue characterization.

\section{References and links}

1. D. Hulmes, "Building collagen molecules, fibrils, and suprafibrillar structures," J. Struct. Biol. 137, 2-10 (2002).

2. J. H. Krachmer, M. J. Mannis, and E. J. Holland, Cornea, 2nd ed. (Mosby, 2005).

3. G. Mosser, A. Anglo, C. Helary, Y. Bouligand, and M.-M. Giraud-Guille, "Dense tissue-like collagen matrices formed in cell-free conditions," Matrix Biol. 25, 3-13 (2006).

4. F. Gobeaux, G. Mosser, A. Anglo, P. Panine, P. Davidson, M.-M. Giraud-Guille, and E. Belamie, "Fibrillogenesis in dense collagen solutions: a physicochemical study," J. Mol. Biol. 376, 1509-1522 (2008).

5. P. De Sa Peixoto, A. Deniset-Besseau, M.-C. Schanne-Klein, and G. Mosser, "Quantitative assessment of collagen I liquid crystal organizations: role of ionic force and acidic solvent, and evidence of new phases," Soft Matter 7, 11203 (2011).

6. P. De Sa Peixoto, A. Deniset-Besseau, M. Schmutz, A. Anglo, C. Illoul, M.-C. Schanne-Klein, and G. Mosser, "Achievement of cornea-like organizations in dense collagen I solutions: clues to the physico-chemistry of cornea morphogenesis," Soft Matter 9, 11241 (2013).

7. M. Schadt, "Liquid crystal materials and liquid crystal displays," Annu. Rev. Mater. Sci. 27, 305-379 (1997).

8. G. W. Gray and P. A. Winsor, Liquid Crystals and Plastic Crystals, vol. 2 (Ellis Horwood Ltd. Publisher, 1974).

9. I. C. Khoo, "Nonlinear optics of liquid crystalline materials," Phys. Rep. 471, 221-267 (2009).

10. D. Yelin, Y. Silberberg, Y. Barad, and J. S. Patel, "Depth-resolved imaging of nematic liquid crystals by thirdharmonic microscopy," Appl. Phys. Lett. 74, 3107-3109 (1999).

11. R. S. Pillai, G. J. Brakenhoff, and M. Müller, "Analysis of the influence of spherical aberration from focusing through a dielectric slab in quantitative nonlinear optical susceptibility measurements using third-harmonic generation," Opt. Express 14, 260 (2006).

12. B. G. Saar, H.-S. Park, X. S. Xie, and O. D. Lavrentovich, "Three-dimensional imaging of chemical bond orientation in liquid crystals by coherent anti- Stokes Raman scattering microscopy," Opt. Express 15, 13585-13596 (2007).

13. A. Deniset-Besseau, P. De Sa Peixoto, G. Mosser, and M.-C. Schanne-Klein, "Nonlinear optical imaging of lyotropic cholesteric liquid crystals," Opt. Express 18, 1113-1121 (2010).

14. M. Iwamoto, F. Liu, and O.-Y. Zhong-Can, "Polarization-dependence of optical second harmonic generation for chiral cylindrical structure and explanation for nonlinear optical imaging of cholesteric liquid crystals," Chem. Phys. Lett. 511, 455-460 (2011).

15. A. Zoumi, A. Yeh, and B. Tromberg, "Imaging cells and extracellular matrix in vivo by using second-harmonic generation and two-photon excited fluorescence," Proc. Natl. Acad. Sci. USA 99, 11014-11019 (2002). 
16. P. Stoller, P. M. Celliers, K. M. Reiser, and A. M. Rubenchik, "Quantitative second-harmonic generation microscopy in collagen," Appl. Opt. 42, 5209-5219 (2003).

17. W. R. Zipfel, R. Williams, R. Christie, A. Nikitin, B. Hyman, and W. Webb, "Live tissue intrinsic emission microscopy using multiphoton-excited native fluorescence and second harmonic generation," Proc. Natl. Acad. Sci. USA 100, 7075-7080 (2003).

18. P. J. Campagnola and L. M. Loew, "Second-harmonic imaging microscopy for visualizing biomolecular arrays in cells, tissues and organisms," Nat. Biotechnol. 21, 1356-1360 (2003).

19. S. V. Plotnikov, A. C. Millard, P. J. Campagnola, and W. A. Mohler, "Characterization of the myosin-based source for second-harmonic generation from muscle sarcomeres," Biophys. J. 90, 693-703 (2006).

20. A. Deniset-Besseau, J. Duboisset, E. Benichou, F. Hache, P.-F. Brevet, and M.-C. Schanne-Klein, "Measurement of the second-order hyperpolarizability of the collagen triple helix and determination of its physical origin," J. Phys. Chem. B 113, 13437-13445 (2009).

21. A. E. Tuer, S. Krouglov, N. Prent, R. Cisek, D. Sandkuijl, K. Yasufuku, B. C. Wilson, and V. Barzda, "Nonlinear optical properties of type I collagen fibers studied by polarization dependent second harmonic generation microscopy,” J. Phys. Chem. B 115, 12759-12769 (2011).

22. C. P. Brown, M. A. Houle, K. Popov, M. Nicklaus, C. A. Couture, M. Laliberte, T. Brabec, A. Ruediger, A. J. Carr, A. J. Price, H. S. Gill, L. Ramunno, and F. Legare, "Imaging and modeling collagen architecture from the nano to micro scale," Biomed. Opt. Express 5, 233-243 (2014).

23. P. Stoller, K. M. Reiser, P. M. Celliers, and A. M. Rubenchik, "Polarization-modulated second harmonic generation in collagen,” Biophys. J. 82, 3330-3342 (2002).

24. F. Tiaho, G. Recher, and D. Rouède, "Estimation of helical angles of myosin and collagen by second harmonic generation imaging microscopy,” Opt. Express 15, 12286-12295 (2007).

25. J. C. Mansfield, C. P. Winlove, J. Moger, and S. J. Matcher, "Collagen fiber arrangement in normal and diseased cartilage studied by polarization sensitive nonlinear microscopy," J. Biomed. Optics 13, 044020 (2008).

26. O. Nadiarnykh and P. J. Campagnola, "Retention of polarization signatures in SHG microscopy of scattering tissues through optical clearing," Opt. Express 17, 5794-5806 (2009).

27. G. Latour, I. Gusachenko, L. Kowalczuk, I. Lamarre, and M.-C. Schanne-Klein, "In vivo structural imaging of the cornea by polarization-resolved second harmonic microscopy," Biomed. Opt. Express 3, 1 (2012).

28. S. Psilodimitrakopoulos, I. Amat-Roldan, P. Loza-Alvarez, and D. Artigas, "Effect of molecular organization on the image histograms of polarization SHG microscopy,” Biomed. Opt. Express 3, 2681-2693 (2012).

29. I. Gusachenko, V. Tran, Y. Goulam Houssen, J.-M. Allain, and M.-C. Schanne-Klein, "Polarization-resolved second-harmonic generation in tendon upon mechanical stretching," Biophys. J. 102, 2220-2229 (2012).

30. J. Duboisset, D. Ait-Belkacem, M. Roche, H. Rigneault, and S. Brasselet, "Generic model of the molecular orientational distribution probed by polarization-resolved second-harmonic generation," Phys. Rev. A 85, 043829 (2012).

31. A. E. Tuer, M. K. Akens, S. Krouglov, D. Sandkuijl, B. C. Wilson, C. M. Whyne, and V. Barzda, "Hierarchical model of fibrillar collagen organization for interpreting the second-order susceptibility tensors in biological tissue," Biophys. J. 103, 2093-2105 (2012).

32. A. Tidu, D. Ghoubay-Benallaoua, B. Lynch, B. Haye, C. Illoul, J.-M. Allain, V. M. Borderie, and G. Mosser, "Development of human corneal epithelium on organized fibrillated transparent collagen matrices synthesized at high concentration," Acta Biomater. 22, 50-58 (2015).

33. C. Teulon, I. Gusachenko, G. Latour, and M.-C. Schanne-Klein, "Theoretical, numerical and experimental study of geometrical parameters that affect anisotropy measurements in polarization-resolved SHG microscopy," Opt. Express 23, 10168-10176 (2015).

34. Y. R. Shen, Principles of Nonlinear Optics (Wiley, 1984).

35. I. Gusachenko, G. Latour, and M.-C. Schanne-Klein, "Polarization-resolved second harmonic microscopy in anisotropic thick tissues," Opt. Express 18, 19339-19352 (2010).

36. H. Lee, M. J. Huttunen, K.-J. Hsu, M. Partanen, G.-Y. Zhuo, M. Kauranen, and S.-W. Chu, "Chiral imaging of collagen by second-harmonic generation circular dichroism," Biomed. Opt. Express 4, 909-916 (2013).

37. M. Rivard, K. Popov, C. A. Couture, M. Laliberte, A. Bertrand-Grenier, F. Martin, H. Pepin, C. P. Pfeffer, C. Brown, L. Ramunno, and F. Legare, "Imaging the noncentrosymmetric structural organization of tendon with interferometric second harmonic generation microscopy,” J. Biophot. 7, 638-646 (2014).

\section{Introduction}

Collagen is the most abundant protein in mammals. It is the main component of connective tissues, including skin, cornea or arteries, and a key element of their architecture. The collagen molecules are triple helices synthesized by cells. Regarding the fibrillar collagen types, these triple helices spontaneously self-assemble into fibrils. Those fibrils further form organized threedimensional (3D) structures specific to each tissue and related to its physical and mechanical properties [1]. In situ 3D visualization at the micrometer scale of this biopolymer is therefore 
a major biomedical concern, whether to study collagen accumulation, impairment or disorder in numerous pathologies, or to decipher the structure of connective tissues and guide tissue engineering.

Dense collagen solutions have been shown to exhibit self organization properties, which can be exploited to synthetize engineered tissues that display the same 3D organization as native tissues, for instance a plywood-like structure similar to the one found in cornea [2]. In particular, it has been shown that acidic solutions of type I collagen form liquid crystals at high concentration [3-6]. These solutions of triple helices may then be stabilized by increasing the $\mathrm{pH}$ to a physiological value, resulting in the formation of collagen fibrils that display the same organization as the liquid solution.

More generally, liquid crystals are widely used in many applications and there is a need for in situ characterization of these materials at the micrometer scale [7]. Optical techniques are particularly relevant for that purpose [8] and may take advantage of the large optical nonlinearities that have been demonstrated in these materials [9]. Nonlinear optical processes such as two-photon excited fluorescence (2PEF), second harmonic generation (SHG), third harmonic generation (THG) or coherent anti-Stokes Raman scattering (CARS) [10-14] have been used to obtain 3D images of liquid crystals at the micrometer scale. Nonlinear optical microscopy indeed provides intrinsic 3D resolution due to the spatial confinement of the nonlinear processes at the focus of the laser excitation.

Among these nonlinear optical techniques, SHG microscopy has been shown to reveal the 3D organization of unstained fibrillar collagen with excellent contrast in biological tissues and in matrices [15-18]. SHG is specific to dense non-centrosymmetric materials. The collagen triple helix exhibits non negligible second order hyperpolarizability due to the presence of numerous tightly aligned peptidic bonds whose nonlinear responses, although quite weak, coherently sum up along the triple helical structure [19-22]. The same coherent summation applies within tightly aligned collagen molecules in fibrils, which explains the large SHG signals of collagen fibrils in tissues. In dense acidic solutions of molecular collagen, the collagen triple helices form liquid crystalline phases, within which triple helices are well aligned at the micrometer scale, allowing detectable SHG signals as in fibrils. Accordingly, we have previously demonstrated nonlinear optical imaging in such collagen liquid crystals $[5,6,13]$.

The aim of this work is to obtain new information about the 3D structure of collagen liquid crystals by using polarization-resolved second-harmonic generation (P-SHG) microscopy. P-SHG has been shown to provide quantitative parameters related to the 3D microstructure of collagen fibrils in biological tissues [23-31]. In this work, P-SHG microscopy is used to characterize nematic plywood-like collagen structures, similar to the ones found in cornea. We demonstrate the benefits of this technique that provides information not accessible with classic imaging modalities, and in particular we highlight its unique sensitivity to the orientation of collagen molecules.

This paper is organized as follows. We first introduce the preparation of dense liquid crystalline collagen samples and the nonlinear imaging setup. Then, we derive an analytical calculation of the P-SHG signal as a function of the 3D orientation of collagen molecules in the excitation volume and connect the measured P-SHG parameters to the liquid crystal orientation. We then report numerical and experimental results for various orientations of the plywood-like structure and show that only P-SHG measurements reveal unambiguously a signature of such structures at specific orientations.

\section{Materials and methods}

\subsection{Sample preparation}

Type I collagen was extracted from rat-tail tendon and purified as previously described $[4,32]$. It was solubilized in $500 \mathrm{mM}$ acetic acid (no fibrils), and then dialyzed against a $10 \mathrm{mM}$ acetic 


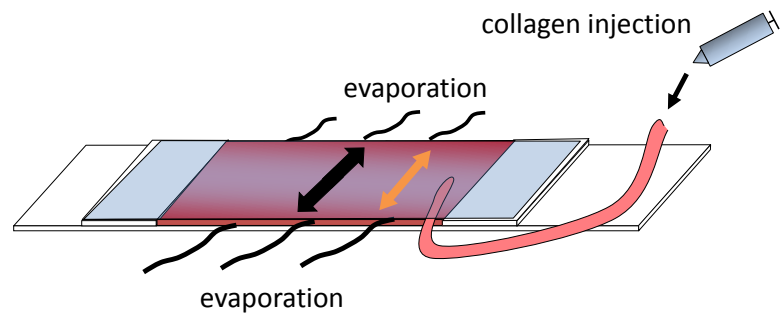

Fig. 1. Customized microchamber allowing collagen concentration. Black arrows: collagen concentration gradient. The concentration is the largest near air-liquid interfaces. Orange arrow: orientation of the plywood director axis near the air-liquid interface (see below).

acid and $0.3 \mathrm{mM}$ hydrochloric acid solution ( $\mathrm{pH}=3.2)$ [32]. The 2 or $3 \mathrm{mg} / \mathrm{mL}$ solution was then concentrated in a two-sided open glass microchamber made out of two coverslips and spacers as depicted in Fig. 1. The thickness of the microchamber was approximately $0.3 \mathrm{~mm}$ over its whole length. Slow and continuous injection of the collagen solution at one end of the microchamber was performed with a syringe during typically 4 days. It aimed at countering solvent evaporation on both open sides and increasing collagen concentration, while creating a concentration gradient near the air-liquid interfaces. This setup has been shown to induce the formation of liquid crystal phases near the air-liquid interface where the collagen concentration is the largest (up to a few hundred $\mathrm{mg} / \mathrm{mL}$ ) [3-5]. All experiments were performed at $21^{\circ} \mathrm{C}$.

The liquid crystalline patterns were dependent on the collagen local concentration and other chemical parameters such as acid strength and concentration, or ionic force [3-6]. In this study, we focussed on a condition giving a plywood like organization, at the micrometric scale, near the liquid-air interface where the collagen concentration was the highest [32].

\subsection{SHG microscopy}

In the present work, we probed the structure of the collagen liquid crystal using SHG signal, and we measured the collagen concentration with 2PEF. For that purpose, we used a custom-built laser scanning nonlinear optical microscope as previously described [33] (see Fig. 2). Excitation was provided by a femtosecond Titanium-Sapphire laser (Mai-Tai, Spectra-Physics) tuned at $860 \mathrm{~nm}$ and scanned in the $X Y$ directions using galvanometric mirrors. We used either circular polarization in order to image all structures independently of their orientation in the image plane, or a set of linear polarizations with different orientations in order to perform polarization-resolved measurements. The orientation of the incident field was controlled by two waveplates inserted at the back aperture of the objective, allowing us to measure the SHG intensity for different laser excitation angles $\theta$. All polarization-resolved images were acquired at 36 excitation angles $\theta$ regularly spaced between $0^{\circ}$ and $360^{\circ}$. We detected simultaneously SHG and 2PEF signals in two detection channels equipped with photon-counting photomultiplier tubes (P25PC, Electron tubes) and suitable spectral filters to reject the excitation beam (FF01-680SP, FF01-720SP, Semrock) and select the proper signal. SHG signals were detected in the trans-direction through a condenser (effective numerical aperture $(\mathrm{NA}) \approx 1$ ) since SHG is mainly forward-directed, using a FF01-427/10 interferential filter (Semrock). 2PEF signals were epidetected through the focusing objective using a GG455 colored filter (Schott). Liquid crystalline samples were imaged in situ using a 60x, 1.2-NA objective lens with coverslip correction (UplanSApo, Olympus), resulting in typically $0.3 \mu \mathrm{m}$ (lateral) x $0.9 \mu \mathrm{m}$ (axial) resolution. Imaging was performed within 2 hours after stopping the injection in order to prevent sample dehydration. Power at the sample was 4 to $10 \mathrm{~mW}$ with 5-10 $\mu \mathrm{s} /$ pixel acquisition time. No perturbation of the texture of the sample was observed in these imaging conditions under continuous excitation, as expected from the 
a.

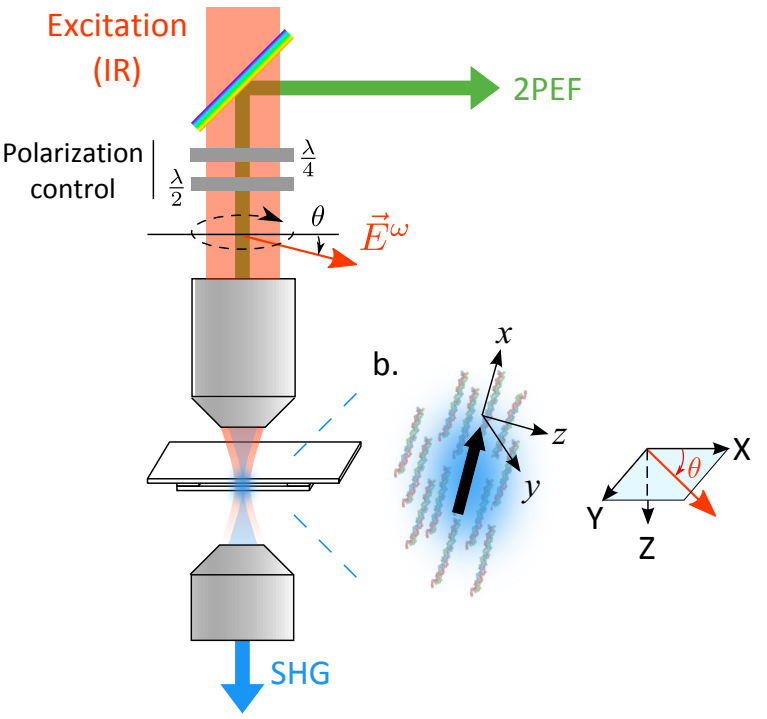

Fig. 2. a. Setup geometry. Excitation polarization angle $\theta$ is controlled by two achromatic waveplates. Excitation field $\vec{E}^{\omega}$ is focused through an objective. $\mathrm{SH}$ radiation is collected forwards through a condensor $(\mathrm{NA} \approx 1)$ and $2 \mathrm{PEF}$ backwards through the focusing objective. b. Zoomed scheme of the excitation volume. The pink arrow gives the mean orientation of all triple helices present in the excitation volume. $(x y z)$ is the corresponding frame, with $x$ along the mean orientation of the triple helices. $(X Y Z)$ is the laboratory frame. Incident polarization $\vec{E}^{\omega}$ is shown in red and is supposed to be in the image plane $(X Y)$.

highly viscous character of the concentrated collagen solutions [13].

\section{Theory}

\subsection{Polarization-resolved SHG microscopy (P-SHG)}

The second harmonic $(\mathrm{SH})$ response of a medium is characterized by its second order nonlinear susceptibility tensor $\chi^{(2)}$ [34], which connects the induced polarizability $\vec{P}^{2 \omega}$ to the incident electric field $\vec{E}^{\omega}$ :

$$
P_{i}^{2 \omega}=\sum_{j, k} \chi_{i j k}^{(2)} E_{j}^{\omega} E_{k}^{\omega}
$$

where $i, j$ and $k$ stand for $x, y$ or $z$ spatial directions in the sample frame (see Fig. 2(b)).

Here, the assembly of collagen triple helices in the excitation volume is supposed to be well aligned together with almost no disorder. We assume that these triple helices exhibit a cylindrical symmetry and that Kleinman symmetry applies since these assumptions have been shown to provide a good agreement with experimental data while simplifying the calculations when using linear polarizations $[16,19,23,24,35]$. Note that lower symmetry including chiral components has to be considered when measuring SHG circular difference [36], which is not the case in this study. Within the cylindrical and Kleinman symmetries, the $\chi^{(2)}$ tensor has only seven non vanishing components with two independent values: $\chi_{x x x}^{(2)}$ and $\chi_{x y y}^{(2)}=\chi_{x z z}^{(2)}=\chi_{y x y}^{(2)}=\chi_{z x z}^{(2)}=\chi_{y y x}^{(2)}=\chi_{z z x}^{(2)}$, in the $(x y z)$ frame where $x$ axis is along the mean orientation of the triple helices. 
a.

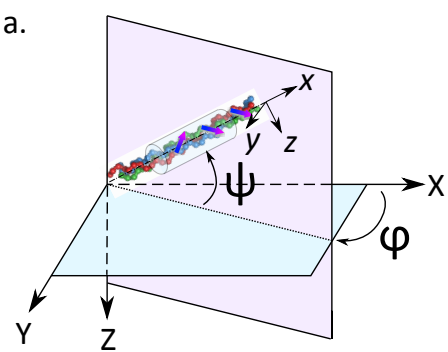

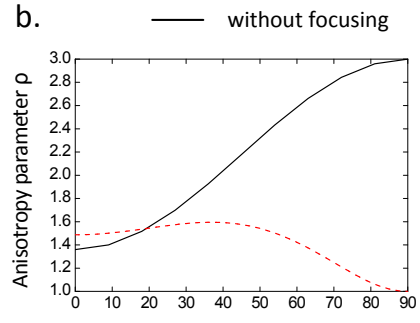

Out of plane angle $\psi\left({ }^{\circ}\right)$

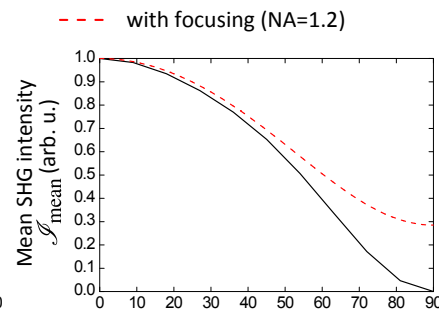

Out of plane angle $\psi\left({ }^{\circ}\right)$

Fig. 3. Collagen orientation. a. Two angles describe the mean $3 \mathrm{D}$ orientation of the triple helices in the excitation volume: $\varphi$, the orientation of its projection in the image plane $(X Y)$ and $\psi$, the out-of-plane angle. b. Evolution of $\mathscr{I}_{\text {mean }}$ and $\rho$ with respect to out-of-plane angle $\psi$, either without focusing (black solid lines, analytic calculations) or with strong focusing and forward detection (red dotted lines, $\mathrm{NA}=1.2$, numerical simulations). $\rho(\psi)$ is plotted for $\rho_{\mathrm{tr}}=1.36$. Note that in the case of strong focusing, those two parameters depend also on geometrical parameters such as focusing NA.

In that case, the polarizability at $2 \omega$ writes [23]:

$$
\begin{aligned}
P_{x}^{2 \omega} & =\chi_{x x x}^{(2)} E_{x}^{2}+\chi_{x y y}^{(2)} E_{y}^{2}+\chi_{x y y}^{(2)} E_{z}^{2}, \\
P_{y}^{2 \omega} & =2 \chi_{x y y}^{(2)} E_{x} E_{y} \\
P_{z}^{2 \omega} & =2 \chi_{x y y}^{(2)} E_{x} E_{z}
\end{aligned}
$$

These equations are written in the reference frame $(x y z)$ attached to the collagen triple helices, while SHG microscopy is performed in the laboratory frame $(X Y Z)$. To switch to the latter frame, we have to describe the mean orientation of the triple helices in the laboratory frame, which is fully obtained by the two angles depicted in Fig. 3(a):

- the orientation $\varphi$ of their projection in the image plane $(X Y)$

- the out-of-plane angle $\psi$

For polarization-resolved measurements in a non focused regime, the incident electric field is perpendicular to the direction of light propagation $Z$ in the laboratory frame $(X Y Z)$ and at angle $\theta$ to the laboratory axis $X$ : its vectorial components are $E_{X}^{\omega}=E_{0} \cos (\theta), E_{Y}^{\omega}=E_{0} \sin (\theta)$ and $E_{Z}^{\omega}=0$. In that case, the total SHG signal intensity is calculated in a straigthforward way by translating the susceptibility tensor from the molecular frame $(x y z)$ to the laboratory frame $(X Y Z)$ (see Appendix). It is then given by [23, 28, 29]:

$$
\mathscr{I}^{2 \omega}(\theta)=\cos ^{2}(\psi) \times[A(\psi) \cos (4(\theta-\varphi))+B(\psi) \cos (2(\theta-\varphi))+C(\psi)]
$$

$A(\psi), B(\psi)$ and $C(\psi)$ are directly related to the susceptibility components in the $(X Y Z)$ frame, which can be written as a function of the susceptibility components of the triple helices in the $(x y z)$ frame and of the out-of-plane angle $\psi$ of their mean orientation (see Appendix).

Three quantitative parameters are then obtained from $\mathscr{I}^{2 \omega}(\theta)$, the SHG intensity measured for different laser polarization angles $\theta$, either with a fit or a Fourier transform algorithm:

- the orientation $\varphi$ of the triple helices projection in the image plane $(X Y)$,

- the mean intensity $\mathscr{I}_{\text {mean }}$, which is directly connected to $\psi$ at first order [13]:

$$
\mathscr{I}_{\text {mean }} \propto \cos ^{2}(\psi)
$$


- the anisotropy parameter $\rho$, which reflects the anisotropy of the nonlinear response of these triple helices [29]: $\rho=\frac{\chi_{X X X}^{(2)}}{\chi_{X Y Y}^{(2)}}$. This parameter is calculated as the square root of the intensity ratio for an excitation field parallel or perpendicular to the mean orientation of the triple helices [29]:

$$
\rho=\sqrt{\frac{\mathscr{I}^{2 \omega}(\varphi)}{\mathscr{I}^{2 \omega}\left(\varphi+\frac{\pi}{2}\right)}}=\sqrt{\frac{A(\psi)+B(\psi)+C(\psi)}{A(\psi)-B(\psi)+C(\psi)}}
$$

It is directly linked to $\psi$ (see Appendix):

$$
\rho=\rho_{\mathrm{tr}} \cos ^{2}(\psi)+3 \sin ^{2}(\psi)
$$

where $\rho_{\mathrm{tr}}=\frac{\chi_{x x x}^{(2)}}{\chi_{x y y}^{(2)}}$ is the ratio obtained for triple helices lying within the image plane $(X Y)$. The ratio $\rho_{\text {tr }}$ depends on the molecular structure of collagen for perfectly aligned triple helices $[19,20,24]$. It varies with the orientation disorder among triple helices in the excitation volume [29]. We used $\rho_{\mathrm{tr}}=1.36$ for analytic calculations [29].

\subsection{Analytic calculations and numerical simulations}

Using the above relationships between anisotropy $\rho$, mean intensity $\mathscr{I}_{\text {mean }}$ and out-of-plane angle $\psi$ and considering that the incident electric field lies in the $(X Y)$ plane (no or slight focusing), it is straightforward to derive analytically P-SHG signals for any direction of the triple helices. These results are presented in Fig. 3(b) (black lines). As expected from equations (3) and (6), the anisotropy parameter increases and the mean intensity decreases when the out-of-plane angle $\psi$ increases.

Nevertheless, high NA objective lens results in a strongly focused incident beam with an axial polarization component. In that case, analytic calculations are not possible. Numerical simulations are needed to derive the pattern of the polarization components of the electric field at focus and to calculate the SHG signal radiated at high NA [33]. Starting from the algorithm presented in [33] that considered only in-plane collagen molecules, modification were included to consider any out-of-plane angle $\psi$. These numerical simulations were performed using a focusing NA 1.2 and for forward detection. For the sake of simplification, we considered homogeneous systems composed of triple helices all aligned along a given direction with out-of-plane angle $\psi$ (no layers). In this way, the two P-SHG parameters $\rho$ and $\mathscr{I}_{\text {mean }}$ were obtained for any angle $\psi$.

Figure 3(b) displays the result of these numerical simulations as a function of the out-of-plane angle $\psi$ (red dotted lines). The behavior of the P-SHG parameters $\rho$ and $\mathscr{I}_{\text {mean }}$ is similar to the one obtained from analytical calculations, but with less contrast. The non zero value of the mean SHG intensity for collagen at right angle from the image plane $\left(\psi=90^{\circ}\right)$ is consistent with the presence of an axial component of the excitation field due to strong focusing. The decrease of the anisotropy parameter at high out-of-plane angles is also attributed to strong focusing, which scrambles the various polarization components and decreases the contrast of P-SHG. Note that, at high angles, the intensity is low, so the anisotropy parameter is determined with low accuracy (see Appendix of [33] for more details).

Since the strong focusing impacts mostly the contrast of the P-SHG parameters we are interested in, we only present in the following the results of analytic calculations while keeping in mind that the observed contrast should be lower. 
a.

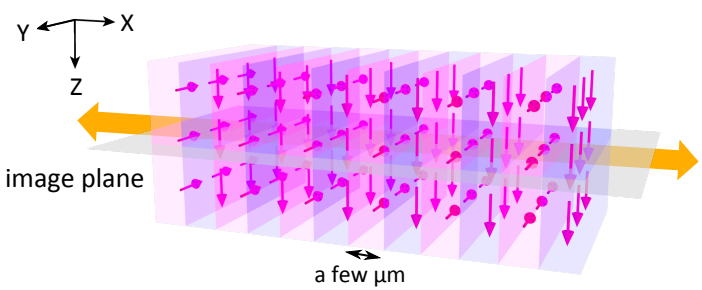

b.

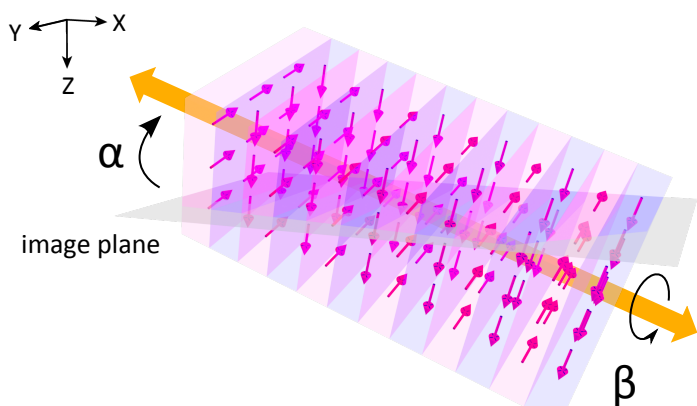

Fig. 4. Nematic plywood-like organization. This structure consists of a few $\mu$ m-thick layers composed of triples helices aligned along a main direction (red arrow). Two sequential layers have orthogonal directions. The director axis (orange arrow) is perpendicular to the layers. We suppose that this axis is in the $(X Z)$ plane to get $\varphi$ angles centered around $90^{\circ}$. a. Simplest case, with the director axis along $X$ axis, and layers directions along $Y$ and $Z$ axis. b. Two angles describe any 3D orientation of this structure: the angle $\alpha$ of the director axis with respect to the image plane and the directions of the layers around this axis, defined by angle $\beta$ ( $\beta=0^{\circ}$ when a layer is along $Y$ axis). See Visualization 1.

\section{Numerical and experimental results}

\subsection{Calculation of P-SHG patterns for plywood-like structures}

The collagen triple helices in the microchamber organize themselves in a liquid crystal phase near the air-liquid interface, where the collagen concentration is the largest. This organization depends on the collagen local concentration and on chemical parameters such as acid nature and concentration, $\mathrm{pH}$ and ionic force [5]. Here, collagen is solubilized in $10 \mathrm{mM}$ acetic acid and 0.3 $\mathrm{mM}$ hydrochloric acid ( $\mathrm{pH}=3.2)$. It results in a nematic plywood-like structure, similar to the one observed for collagen fibrils in cornea and depicted in Fig. 4 [6]. This liquid crystal phase is composed of layers having a thickness of a few $\mu \mathrm{m}$ and containing aligned triple helices along a given direction (red arrow). Two sequential layers are expected to have perpendicular directions. The axis around which the layer directions turn is the director axis (orange arrow). For the sake of simplification, we suppose here that it lies in the $(X Z)$ plane. Therefore, only two parameters are required to describe this liquid crystal phase in the (XYZ) fixed frame (Fig. 4(b)):

- the angle $\alpha$ of this director axis with respect to the image plane $(X Y)$,

- the two possible layer directions, which are described by only one angle $\beta$ since they are perpendicular. We set $\beta=0^{\circ}$ when a layer is oriented along $Y$ axis. We consider that collagen triple helices have the same polarity within a layer, but we do not take explicitly this polarity into account, so that $\beta$ ranges from $0^{\circ}$ to $90^{\circ}$ here. In-plane polarity may be measured using interferometric SHG microscopy in a thin sample [37], but we are interested in the collagen 3D organization of thick samples in this study. 


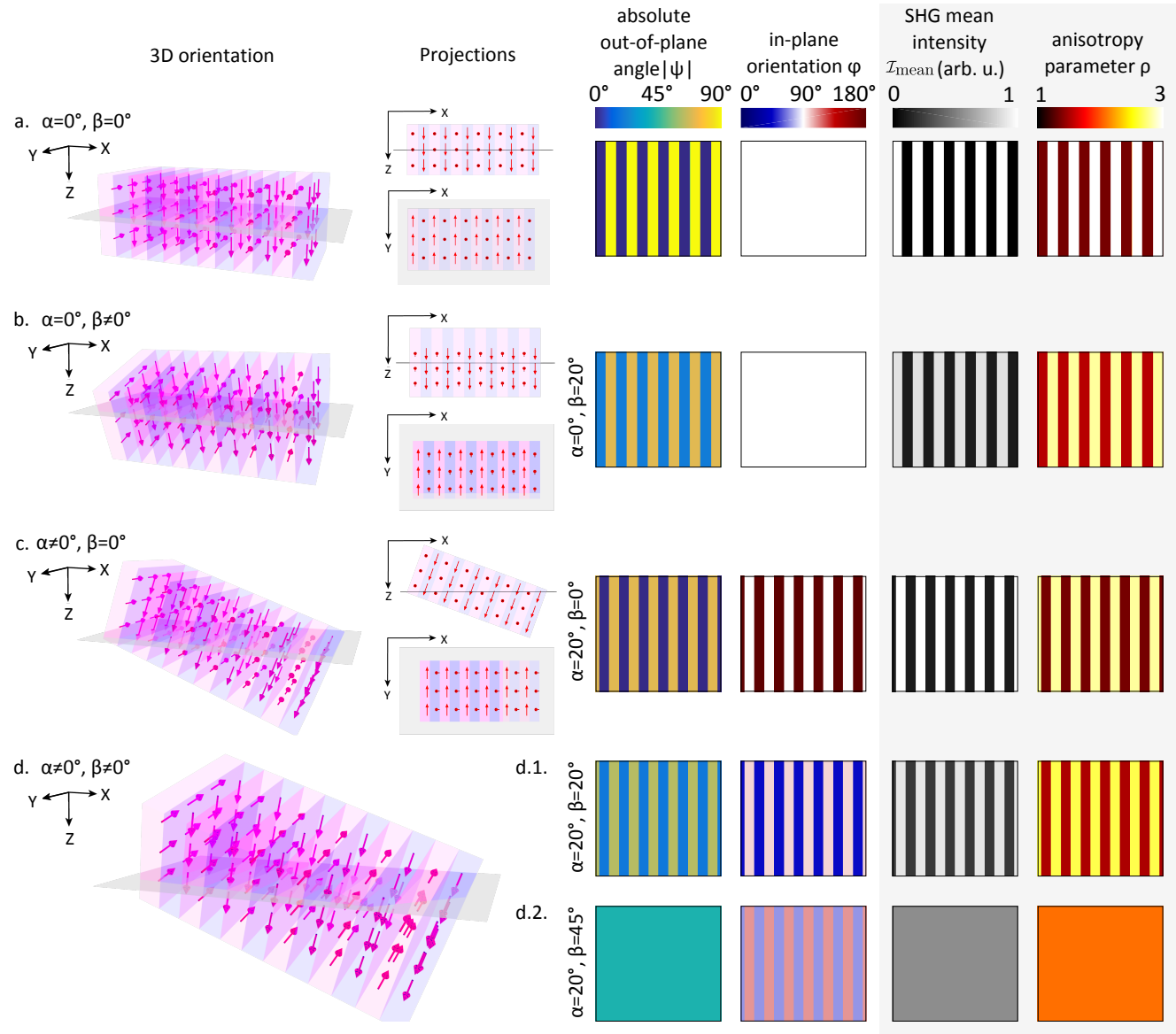

Fig. 5. Theoretical P-SHG patterns obtained from analytic calculations for different 3D orientations of the plywood-like structure: $\mathbf{a} . \alpha=0^{\circ}$ and $\beta=0^{\circ}$, b. $\alpha=0^{\circ}$ and $\beta=20^{\circ}$, c. $\alpha=20^{\circ}$ and $\beta=0^{\circ}$, d.1. $\alpha=20^{\circ}$ and $\beta=20^{\circ}$, d.2. particular case $\alpha=20^{\circ}$ and $\beta=45^{\circ}$. For the three first orientations, the projections in the image plane $(X Y)$, in gray, and in the $(X Z)$ plane are shown. For the two last ones, the projections of two different layers are not along the same plane and cannot be displayed. Orientation $\varphi$ is defined here with respect to $X$ axis. Comparison of the 3D orientations corresponding to the same angle $\alpha$ : a and $\mathrm{b}$, or $\mathrm{c}$ and $\mathrm{d}$, show that the three P-SHG parameters have less contrast when $\beta \neq 0^{\circ}$. See Visualization 1. 
P-SHG measurements then depend on the orientation of the triple helices in the laboratory frame $(X Y Z)$, described by $(\varphi, \psi)$, ie on how the plywood frame, described by $(\alpha, \beta)$ is oriented with respect to $(X Y Z)$. The plywood-like structure can be oriented in any direction with respect to the air-liquid interface. In practice, the layers of the plywood-like structure are roughly parallel to the air-liquid interface, but can be a bit tilted with respect to this interface. We indeed observe in the in-depth 3D images a small tilt angle $\alpha$ of the director axis with respect to the image plane.

We performed analytic calculations using equations derived in section 3.1, to connect the three measured P-SHG parameters $\mathscr{I}_{\text {mean }}, \rho$ and $\varphi$ to the plywood 3D orientation $(\alpha, \beta)$, in the case of no focusing. Results are displayed in Fig. 5 and in the movie S1, for four different cases:

$\alpha=0^{\circ}$ and $\beta=0^{\circ}$ (Fig. 5(a)) In this simplest case, triple helices are either in the image plane or perpendicular to it. Analytic calculations show that:

- the orientation in the image plane $\varphi$ is the same everywhere

- the mean intensity exhibits fringes: the maxima $\left(\mathscr{I}_{\text {mean }}=1\right)$ correspond to layers where triple helices are in-plane and the minima $\left(\mathscr{I}_{\text {mean }}=0\right)$ to the other layers (perpendicular to the image plane).

- the anisotropy parameter $\rho$ shows a similar behavior: $\rho=\rho_{\text {tr }}=1.36$ when the triple helices are in plane, and $\rho=3$ when they are perpendicular to the image plane.

$\alpha=0^{\circ}$ and $\beta \neq 0^{\circ}$ (Fig. 5(b)) We observe the same kind of images, with less contrast: $\rho$ switches from 1.55 to 2.81 and $\mathscr{I}_{\text {mean }}$ from 0.12 to 0.88 .

$\alpha \neq 0^{\circ}$ and $\beta=0^{\circ}$ (Fig. 5(c)) Analytic calculations show that:

- the mean intensity exhibits fringes as in the former cases, but with lower contrast.

- the anisotropy $\rho$ exhibits the same kind of behavior, with lower contrast.

- the orientation in the image plane $\varphi$ also oscillates.

$\alpha \neq 0^{\circ}$ and $\beta \neq 0^{\circ}$ (Fig. 5(d)) We observe the same kind of images, with less contrast (Fig. 5(d.1)). In the particular case $\beta=45^{\circ}$ (Fig. 5(d.2)), $\rho$ and $\mathscr{I}_{\text {mean }}$ exhibit no contrast at all, because triple helices have the same out-of-plane angle $\psi$ in all layers. Here, the variation of $\varphi$, obtained though polarization resolution, is necessary to spot the 3D organization.

In case of strong focusing, we expect that the contrast of $\rho$ and $\mathscr{I}_{\text {mean }}$ is even lower, as it was previously shown in Fig. 3(b). The expected geometry corresponds to cases (c) and (d).

\subsection{Experimental results}

We recorded P-SHG images in 7 different microchambers with the same physico-chemical conditions $(10 \mathrm{mM}$ acetic acid and $0.3 \mathrm{mM}$ hydrochloric acid solution - $\mathrm{pH}=3.2)$ leading to a nematic plywood-like organization. Collagen density was measured using $2 \mathrm{PEF}$ with the same protocol as described in [13]: it ranged between 300 and $1000 \mathrm{mg} / \mathrm{mL}$ in the observed areas and it was homogeneous in the field of view. Measurements were reproducible in all tested microchambers with those physico-chemical conditions. We removed images from other microchambers exhibiting the following well identified experimental problems: (i) the solution had dryed in the microchamber in case of collagen injection for a too long time; (ii) the collagen didn't achieve liquid crystal organization because the collagen density was too low; (iii) the liquid crystal organization was disrupted because the microchamber was shocked or broken.

Figure 6 displays the three parameters of interest obtained from P-SHG measurements in two typical microchambers. Figures 6(a) and 6(b) display those parameters in the imaged area for each microchamber. A zoom of the yellow squared area (Fig. 6(b)) is shown in Fig. 6(c), 
and the profiles of each parameter in a direction perpendicular to the fringes (yellow line) are displayed in Fig. 6(d). The same pattern was observed in all microchambers, with different levels of contrast: the measured orientation in the image plane $\varphi$ varies periodically (Figs. 6(a.3), 6(b.3) and 6(c.3)), and consistent patterns are observed in the three corresponding images of the same area (zoom in Fig. 6(c)) and in the profiles (Fig. 6(d)). The profiles show that the variations of the SHG mean intensity (Fig. 6(d.1)) and the ones of the anisotropy parameter (Fig. 6(d.2)) are correlated.

At first glance, this pattern is consistent with analytic calculations shown on the right side of Fig. 5. The mean intensity and the anisotropy parameter have the same periodicity, which is consistent with the fact that they depend on the same parameter $\psi$. Furthermore, as expected from analytic calculations and numerical simulations (Fig. 3(b)), when the intensity is high, the anisotropy parameter is low (triple helices in the image plane) and the other way around (triple helices perpendicular to the image plane).

Moreover, we used the data displayed in Fig. 3(b) to tentatively extract the absolute value of the out-of-plane angle $\psi$ from intensity $\mathscr{I}_{\text {mean }}$ and anisotropy parameters $\rho$ measurements. We fitted the simulated data in strong focusing conditions to obtain 2 calibration curves for the anisotropy parameter and the SHG intensity. The one for the anisotropy parameter provided 2 absolute values. Its ambiguity was lifted by the relative value of the SHG intensity. The resulting profile is shown in Fig. 6(e). It is only qualitative because of various uncertainties in experimental conditions, such as inhomogeneities and disorder in the sample, and related slight variations of excitation intensity at focus. Nevertheless, it shows unambiguously that the out-of-plane angle $\psi$ oscillates between two different values, indicating that the orientation of the triple helices is indeed turning with respect to the image plane.

\section{Discussion}

In this study, we explored the benefits of P-SHG microscopy to probe the 3D orientation of collagen molecules. We performed analytical calculations in a non focusing regime and numerical simulations with strong focusing to analyse P-SHG images of liquid crystal organized collagen structures. Experimental and theoretical results are in good agreement, as clearly shown in the zoomed-in images and the profiles in Figs. 6(c) and 6(d), which exhibit fringes similar to the ones obtained by the theoretical calculations displayed in Fig. 5.

Nevertheless, the experimental fringes are not as regular and abrupt as the theoretical ones. In practice, experimental intensity profiles show rather series of Lorentzian peaks than the simulated bright and dark fringes (Fig. 6(d)). This can be explained by some limitations of the theoretical calculations, which consider homogeneous media with aligned triple helices all pointing in the same direction, and then let this direction vary to obtain data at different orientations. In contrast, experiments are performed in a liquid crystal composed of layers with different orientations. Numerical simulations accounting for the actual organization in layers, with frontiers, would be highly complex and are beyond the scope of this paper. We can however anticipate that it may affect the P-SHG data in the following ways:

- The excitation field may be modified while traveling across the liquid crystal, resulting in a slightly disrupted excitation pattern at focus (polarization, phase and intensity);

- At the frontier between two layers, the excitation volume $(\approx 1 \mu \mathrm{m})$ may contain triple helices with different orientations;

- This frontier may be tilted with respect to the image plane;

- The frontiers between two layers may not be infinitely small as in the calculations: in that case, these frontier regions may be considered as disordered regions, with various 3D orientations of the triple helices . 


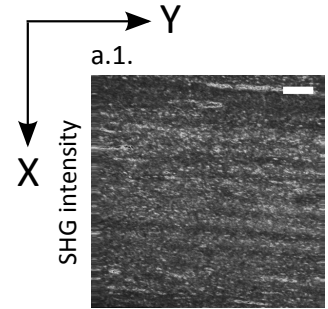

$$
\text { a.2. }
$$
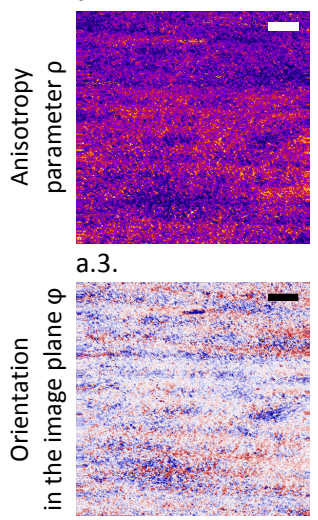

b.1.

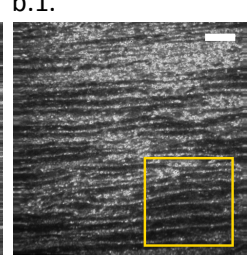

b.2.

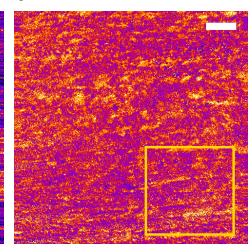

b.3.

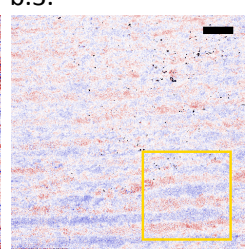

c.1.

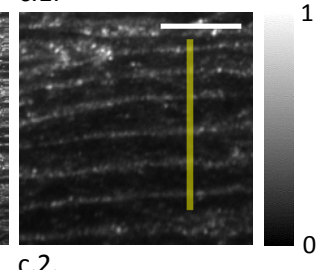

c.2.

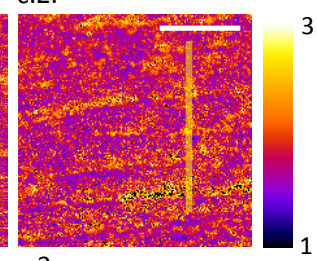

c.3.

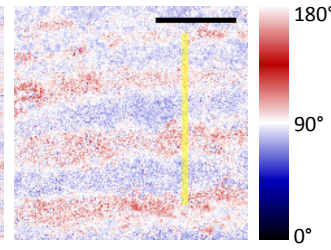

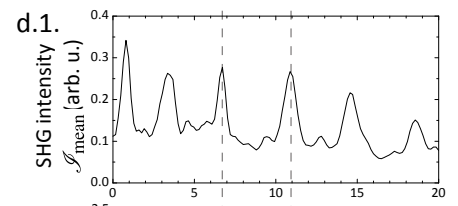

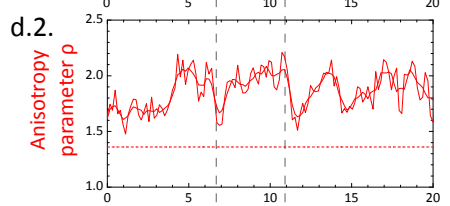

d.3.
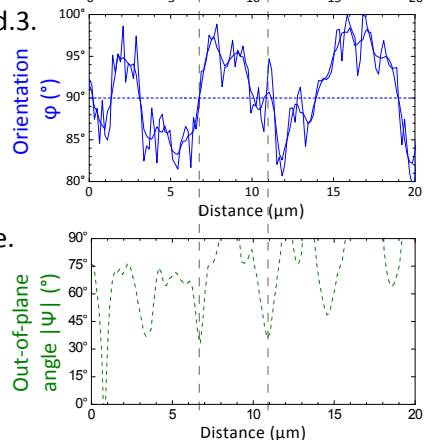

Fig. 6. P-SHG imaging of collagen liquid crystals. a. and b. Set of images obtained from P-SHG measurements in two different microchambers with the same physico-chemical conditions (10mM acetic acid and $0.3 \mathrm{mM}$ hydrochloric acid solution) leading to a nematic plywood-like organization. Collagen density was measured with $2 \mathrm{PEF}$ and ranges between 300 and $1000 \mathrm{mg} / \mathrm{mL}$ in the observed areas. Scalebar: $10 \mu \mathrm{m}$. c. Zoomed-in images taken in the yellow squared areas in b. Same scalebar. d. Transverse profiles of the three P-SHG parameters along the yellow lines in c. e. Absolute value of the out-of-plane angle $\psi$ tentatively deduced from $\mathscr{I}_{\text {mean }}$ and $\rho$. The observed switch between 2 orientations shows that triple helices are indeed rotating around the director axis.

All these effects result in wider and smoother transitions between aligned domains in the image (a few $\mu \mathrm{m}$ ). Moreover, the frontiers correspond to disorder in the excitation volume, which decreases the SHG intensity and increases the anisotropy parameter $\rho$, forming the plateaus observed in the images. Taking these frontier regions into account, we therefore expect from analytic calculations that (i) the in-plane angle $\varphi$ is $90^{\circ}$ when the intensity is maximum (because the triple helices are perpendicular to the director axis, that is to $X$, when $\psi=0^{\circ}$ ), (ii) the in-plane angle $\varphi$ exhibits values over or below $90^{\circ}$ depending on the way the direction of the triple helices switches from one layer to the next one. This is indeed observed in the experimental profiles in Fig. 6(d). Note that the range of variation of this angle $\varphi$ is slightly smaller than the one in the simulated profiles because the tilt angle of the liquid crystal may be smaller than the one considered for the simulations or because the frontiers regions may attenuate the variation of the measured angle.

The out-of-plane angle $\psi$ profile (Fig. 6(e)) confirms the expected and measured variations of $\rho, \mathscr{I}_{\text {mean }}$ and $\varphi$. Indeed, at frontiers between two layers, when the intensity is maximum and $\rho$ drops ( $\varphi$ is then equal to $\left.90^{\circ}\right), \psi$ drops down to zero since triple helices are expected to be in the image plane.

Taking those artifacts into account, we attribute the observed pattern in our experimental data to a plywood-like structure oriented like in case (c) or (d) of Fig. 5. The three measured P-SHG parameters in Figs. 6(b) and 6(c) indeed show fringes similar to the ones calculated for these 
cases in Fig. 5. This structure is confirmed by the 3D intensity images in depth, even if the angle $\varphi$ does not vary with the expected period (Figs. 6(b.3) and 6(c.3)).

Moreover, this study demonstrates that P-SHG reveals features that are otherwise not detected. Indeed, in the images depicted in Fig. 6(a), SHG mean intensity (Fig. 6(a.1)) and anisotropy parameter (Fig. 6(a.2)) patterns do not exhibit enough contrast to observe the fringes specific to a plywood-like organized structure. SHG images recorded with circularly polarized excitation would exhibit the same limitation since they are equivalent to the mean SHG intensity $\mathscr{I}_{\text {mean }}$ in P-SHG measurements. In contrast, the orientation $\varphi$ obtained from P-SHG measurements shows variations (Fig. 6(a.3)). We are in the particular case depicted in Fig. 5(d.2), where P-SHG is needed to spot an organization. In this case, the classical implementation of SHG microscopy may miss the signature of some organized structures, such as a plywood-like structures which are a bit tilted with respect to the image plane. It is especially true for strongly focused excitation because of polarization scrambling.

\section{Conclusion}

In conclusion, our study demonstrates th at P-SHG measurements allow reliable characterization of organized liquid crystal phases by measuring complementary parameters connected to the $3 \mathrm{D}$ orientation of the molecules, and in particular by mapping their in-plane orientation $\varphi$ with sub-micrometer accuracy. This method can be used to establish phase diagrams of the collagen solutions in given physico-chemical conditions. To that end, image pattern of the complementary parameters obtained by P-SHG can be quantitatively linked to the collagen concentration that is measured by use of $2 \mathrm{PEF}$ imaging. In particular, the period of the structure, notably in the in-plane orientation $\varphi$ image, and the critical collagen concentration at the transition between organized and disordered structures can be measured, which is not possible using other techniques. P-SHG measurements should therefore help to optimize physico-chemical conditions leading to a highly organized collagen 3D structure similar to the one found in native corneas, in order to engineer corneal implants which display appropriate biomechanical and biophysical properties. This method may also be generalized to characterize other distributions of polymers, for instance cholesteric collagen structures in bone or cellulose's organization in plants.

\section{Appendix: derivation of the P-SHG response for tilted molecules}

In this section, we derive the P-SHG response for tilted collagen molecules. The SHG response of collagen is known in the frame $(x y z)$ of the triple helices, using Eq. 1, and it has to be translated in the laboratory frame $(X Y Z)$ [34]. Indeed, the incident electric field lies in the $(X Y)$ plane, at an angle $\theta$ to the $X$ axis (see Fig. 2(a)), so that its vectorial components are expressed in the laboratory frame $(X Y Z)$ as follows (considering a non-focusing regime):

$$
E_{X}^{\omega}=E_{0} \cos (\theta), E_{Y}^{\omega}=E_{0} \sin (\theta) \text { and } E_{Z}^{\omega}=0
$$

We use the Euler matrix $\mathrm{M}$ to describe the change of coordinates from the triple helices frame to the laboratory frame:

$$
M=\left[\begin{array}{ccc}
\cos \varphi \cos \psi & -\sin \varphi & \cos \varphi \sin \psi \\
\sin \varphi \cos \psi & \cos \varphi & \sin \varphi \sin \psi \\
-\sin \psi & 0 & \cos \psi
\end{array}\right]
$$

where the angles $\theta$ and $\varphi$ are defined in Fig. 3(a). In order to simplify this matrix, we set $\varphi=0^{\circ}$ and get the proper in-plane orientation by replacing $\theta$ by $\theta-\varphi$. In that case,

$$
\mathrm{M}=\left[\begin{array}{ccc}
\cos \psi & 0 & \sin \psi \\
0 & 1 & 0 \\
-\sin \psi & 0 & \cos \psi
\end{array}\right]
$$




\section{Optics EXPRESS}

and $E_{X}^{\omega}=E_{0} \cos (\theta-\varphi), E_{Y}^{\omega}=E_{0} \sin (\theta-\varphi)$ and $E_{Z}^{\omega}=0$.

The tensor $\chi^{(2)^{\prime}}$ connecting $P_{X, Y, Z}^{2 \omega}$ and $E_{X, Y, Z}^{\omega}$ in the laboratory frame is then calculated as follows:

$$
\chi_{I J K}^{(2)^{\prime}}=\sum_{i, j, k} \mathrm{M}_{I, i} \mathrm{M}_{J, j} \mathrm{M}_{K, k} \chi_{i j k}^{(2)}
$$

We obtain only two independent values for the non zero components for in-plane excitation and detection:

$$
\begin{aligned}
\chi_{X X X}^{(2)} & =\cos \psi\left(\chi_{x x x}^{(2)} \cos ^{2} \psi+3 \chi_{x y y}^{(2)} \sin ^{2} \psi\right) \\
\chi_{X Y Y}^{(2)}=\chi_{Y X Y}^{(2)}=\chi_{Y Y X}^{(2)} & =\cos \psi \chi_{x y y}^{(2)}
\end{aligned}
$$

$P_{X, Y}^{2 \omega}$ then writes:

$$
\begin{aligned}
& P_{X}^{2 \omega}=E_{0}^{2}\left(\chi_{X X X}^{(2)} \cos ^{2}(\theta-\varphi)+\chi_{X Y Y}^{(2)} \sin ^{2}(\theta-\varphi)\right) \\
& P_{Y}^{2 \omega}=2 E_{0}^{2} \chi_{Y Y X}^{(2)} \cos (\theta-\varphi) \sin (\theta-\varphi)
\end{aligned}
$$

Finally, the total SHG signal intensity is obtained as:

$$
\mathscr{I}^{2 \omega}(\theta) \propto\left|P_{X}^{2 \omega}\right|^{2}+\left|P_{Y}^{2 \omega}\right|^{2}
$$

It writes:

$$
\begin{aligned}
\mathscr{I}^{2 \omega}(\theta) & \propto I_{0}^{2}\left[\frac{\left(\chi_{X X X}^{(2)}-\chi_{X Y Y}^{(2)}\right)^{2}}{8}-\frac{\left(\chi_{X Y Y}^{(2)}\right)^{2}}{2}\right] \cos (4(\theta-\varphi)) \\
& +I_{0}^{2}\left[\frac{\left(\chi_{X X X}^{(2)}-\chi_{X Y Y}^{(2)}\right)\left(\chi_{X X X}^{(2)}+\chi_{X Y Y}^{(2)}\right)}{2}\right] \cos (2(\theta-\varphi)) \\
& +I_{0}^{2}\left[\frac{\left(\chi_{X X X}^{(2)}+\chi_{X Y Y}^{(2)}\right)^{2}}{4}+\frac{\left(\chi_{X X X}^{(2)}-\chi_{X Y Y}^{(2)}\right)^{2}}{8}-\frac{\left(\chi_{X Y Y}^{(2)}\right)^{2}}{2}\right]
\end{aligned}
$$

We now define $\rho=\frac{\chi_{X X X}^{(2)}}{\chi_{X Y Y}^{(2)}}$ in the $(X Y Z)$ frame and $\rho_{\text {tr }}=\frac{\chi_{x x x}^{(2)}}{\chi_{x y y}^{(2)}}$ in the $(x y z)$ frame. From Eq. (11), we get:

$$
\rho=\rho_{\mathrm{tr}} \cos ^{2}(\psi)+3 \sin ^{2}(\psi)
$$

Then,

$$
\begin{aligned}
\mathscr{I}^{2 \omega}(\theta) & \propto\left(\chi_{X Y Y}^{(2)}\right)^{2} I_{0}^{2}\left[\frac{(\rho-1)^{2}}{8}-\frac{1}{2}\right] \cos (4(\theta-\varphi)) \\
& +\left(\chi_{X Y Y}^{(2)}\right)^{2} I_{0}^{2}\left[\frac{(\rho-1)(\rho+1)}{2}\right] \cos (2(\theta-\varphi)) \\
& +\left(\chi_{X Y Y}^{(2)}\right)^{2} I_{0}^{2}\left[\frac{(\rho+1)^{2}}{4}+\frac{(\rho-1)^{2}}{8}+\frac{1}{2}\right]
\end{aligned}
$$

so that $\mathscr{I}^{2 \omega}(\theta)$ can be written as in Eq. (3):

$$
\mathscr{I}^{2 \omega}(\theta)=\cos ^{2}(\psi) \times[A(\psi) \cos (4(\theta-\varphi))+B(\psi) \cos (2(\theta-\varphi))+C(\psi)]
$$

with

$$
\begin{array}{r}
A(\psi)=K\left(\chi_{x y y}^{(2)}\right)^{2} I_{0}^{2}\left[\frac{(\rho-1)^{2}}{8}-\frac{1}{2}\right] \\
B(\psi)=K\left(\chi_{x y y}^{(2)}\right)^{2} I_{0}^{2}\left[\frac{(\rho-1)(\rho+1)}{2}\right] \\
C(\psi)=K\left(\chi_{x y y}^{(2)}\right)^{2} I_{0}^{2}\left[\frac{(\rho+1)^{2}}{4}+\frac{(\rho-1)^{2}}{8}+\frac{1}{2}\right]
\end{array}
$$




\section{Optics EXPRESS}

$\rho$ is then obtained from these parameters as in Eq. (5).

$A(\psi), B(\psi)$ and $C(\psi)$ depend on the susceptibility components for a triple helix in the $(x y z)$ frame, on the disorder among triple helices in the excitation volume, and on their mean out of plane angle $\psi$ (see Eq. (6)).

Finally, the mean intensity writes at first order [13]:

$$
\mathscr{I}_{\text {mean }}=C(\psi) \cos ^{2}(\psi)
$$

so that it is proportional to $\cos ^{2}(\psi)$.

\section{Acknowledgments}

The authors gratefully acknowledge B. Binet from the Centre-Poly-Media of Ecole Polytechnique for the 3D schemes in Fig. 4 and 5 and for the movie in the supplementary information, $\mathrm{X}$. Solinas and J.-M. Sintès for technical implementation of the setup and G. Latour for critical reading of the manuscript. This work was partly supported by Agence Nationale de la Recherche (contracts ANR-10-INBS-04 France BioImaging and ANR-11-EQPX-0029 Morphoscope2). C. T. was supported by a grant "Contrat Doctoral Spécifique Normalien” from MESR. G.M. and A.T. thank the Fondation pour la Recherche Médicale for the Grant No DCM20121225759. F.P. was supported by a grant from Ecole Doctorale 397. 